Farrand A, Hosey G \& Buchanan-Smith HM (2014) The visitor effect in petting zoo-housed animals: Aversive or enriching?, Applied Animal Behaviour Science, 151, pp. 117-127.

This is the peer reviewed version of this article

NOTICE: this is the author's version of a work that was accepted for publication in Applied Animal Behaviour Science. Changes resulting from the publishing process, such as peer review, editing, corrections, structural formatting, and other quality control mechanisms may not be reflected in this document. Changes may have been made to this work since it was submitted for publication. A definitive version was subsequently published in Applied Animal Behaviour Science, [VOL 151 (2014)] DOI:

http://dx.doi.org/10.1016/j.applanim.2013.11.012 
Please contact h.m.buchanan-smith@stir.ac.uk directly if you would like a personal copy of the published article. Applied Animal Behaviour Science 151 (2014), 117-127

\section{The Visitor Effect in Petting Zoo-housed Animals: Aversive or Enriching?}

Alexandra Farrand ${ }^{\mathrm{a}}$, Geoff Hosey ${ }^{\mathrm{b}}$ and Hannah M. Buchanan-Smith ${ }^{\mathrm{a}^{*}}$

${ }^{a}$ Psychology, School of Natural Sciences, University of Stirling, Stirling, Scotland, FK9 4LA, United Kingdom

${ }^{\mathrm{b}}$ Biology, University of Bolton, Deane Road, Bolton BL3 5AB, United Kingdom

Email address: gh2@bolton.ac.uk

* Corresponding author. Psychology, School of Natural Sciences, University of Stirling, Stirling, Scotland, FK9 4LA, United Kingdom

Email address: $\underline{\text { h.m.buchanan-smith@stir.ac.uk }}$

Telephone: +44 1786467674

Key words: visitor effect; petting zoo; ungulate; animal welfare; enrichment; human-animal interaction 


\begin{abstract}
Two studies were carried out on the effect of visitors on mixed-breed goats, llama, and Vietnamese pot-bellied pigs housed in a petting zoo display within a safari park. In the first study we investigated the effect of the presence and density of visitors on the animals' behaviour and in the second, the effect of animal grooming by the visitors. We hypothesised that the animals' behaviour would be negatively affected by presence of visitors compared to periods of their absence, but this hypothesis was not fully supported by the data. Goat and llama behaviour was unaffected by the presence of the public, while the pigs showed decreased inactivity and social behaviour, both affiliative and aggressive, when visitors were present.
\end{abstract}

All three study species exhibited increased levels of non-aggressive interaction with the public when visitor density was higher but the level of avoidance or aggression towards visitors was not dependent on density. The goats were less often in physical contact with other goats and less likely to be within proximity of a non-conspecific when visitor numbers were high, whereas the pigs showed decreased feeding, a behaviour that constituted a majority of their activity budget. Species differences were observed in the proportion of samples the study groups interacted in a non-aggressive manner with visitors, goats being most likely and llama the least likely to engage in this behaviour. In the second study visitors were provided with a grooming tool and asked to groom the animals, but no significant behavioural change was observed in either goats or pigs as a result. Visitors groomed goats, but not pigs, more than they interacted with them in non-grooming interactions, and goats, but not pigs, responded less to grooming as visitor density increased.

Although significant behavioural changes in all three study species were associated with either visitor presence or density, the low levels of avoidance of visitors, visitor-directed aggression, or animaldirected aggression, suggest the welfare of the study animals was not profoundly impacted by visitorrelated stimuli. Furthermore, there was no evidence that grooming by the public was enriching for the animals. The species differences reported here do suggest, however, that Vietnamese pot-bellied pigs and 
llama are more sensitive to visitor pressure than goats and particular attention to their welfare may be necessary when they are housed in petting zoo displays.

\section{Introduction}

The petting zoo is usually an exhibit or collection of exhibits where a selection of domesticated or semi-domesticated species are allowed to come into close proximity with humans, often with direct physical contact and sanctioned feeding. Such close contact and interactions have the potential to influence the behaviour and welfare of the animals housed in this type of zoo exhibit. Although petting zoos are popular as stand-alone attractions or within zoos/wildlife parks, they have received little study. An exception is research which suggests visitors negatively influence the behaviour of goats and sheep in a petting zoo (Anderson et al., 2002, 2004). In an agricultural context, however, human contact and handling appear to be beneficial to these animals, resulting in less fearful behaviour and positive physiological changes (Jackson and Hackett, 2007, Markowitz et al., 1998, Rushen et al., 1999), and the possibility exists that similar changes could be brought about in petting zoos, which often contain domesticated farm animals.

Zoo visitors have been associated with behavioural change in captive animals, most notably primates (Hosey, 2000). While visitors can hypothetically be a negative, neutral, or positive influence on zoo animals (Hosey, 2000), the most common behavioural changes reported are generally interpreted as negative in terms of animal welfare. These include decreased social behaviour (Chamove et al., 1988, Glatston et al., 1984, Mallapur et al., 2005, Wood, 1998), increased abnormal behaviour (Blaney and Wells, 2004, Chamove et al., 1988, Mallapur and Chellam, 2002, Mallapur et al., 2005, Skyner et al., 2004, Wells, 2005) and increased aggression (Blaney and Wells, 2004, Chamove et al.,1 988, Glatston et al., 1984, Kuhar, 2008, Mitchell et al., 1991, Wells, 2005). More recently, physiological changes indicative of decreased welfare have been reported in relationship to visitor-related variables in spider monkeys (Ateles geoffroyii rufiventris; Davis et al., 2005) and black rhinoceros (Dicornis bicornis; Carlstead and Brown, 2005). 
Visitor effect research has often lacked scientific rigorousness because of the difficulty controlling variables related to visitors, such as visitor presence, visitor density, visitor noise, and visitor behaviour. Despite methodological concerns, researchers have compared the behaviour of captive animals in the presence of visitors to periods in which visitors are absent, but achieving a condition in which there are no visitors present is difficult in many zoos. For example, twelve ungulate species were found to be more vigilant toward keepers when visitors were absent (Thompson, 1989), but this study included data collected after the zoo had closed to visitors for the day, which is not ideal as it introduces time of day confounds.

Visitor effect research has also been conducted at zoos that are not open every day of the week, allowing observations to be made on days with visitors present and days when visitors are absent; this methodology is acceptable when investigating the short-term visitor effect but is less useful when attempting to identify the chronic effect of zoo visitors (Mallapur et al., 2005). On days when visitors were not present, lion-tailed macaques (Macaca silenus) were observed to decrease self-directed behaviour, increase social and reproductive behaviour, and decrease use of the front of their enclosures (Mallapur et al., 2005), interpreted as signs of improved welfare. Decreased resting has been identified in Indian leopards (Panthera pardus fusca; Mallapur and Chellam, 2002), but increased resting and affiliation and decreased feeding has been reported in green monkeys (Cercopithecus aethiops sabaeus; Fa, 1989) on days when the study zoos were closed to visitors, demonstrating some of the difficulty in interpreting behavioural measures of visitor impact. A more extended period of time (six weeks) without visitors was achieved opportunistically during the outbreak of foot and mouth disease in the United Kingdom in 2001 and Davis et al. (2005) found that spider monkey urinary cortisol was lower in this period than when the zoo was open to the public, although one must consider that the possibility that institutional changes brought about by this outbreak, such as altered husbandry practices or a reduction of staff to only essential personnel, may have affected the results of this study.

Moving study animals from on-exhibit enclosures to off-exhibit enclosures has been employed to change the levels of visitor exposure, but environmental changes in housing conditions, aside from the 
presence of visitors, limit the usefulness of such data. Improvements in lion-tailed macaque welfare (decreased abnormal and stereotypic behaviour), were achieved moving on-display groups to off-display enclosures (Mallapur et al., 2005). However, not all studies have found positive effects; cotton-top tamarins (Saguinus oedipus) showed less social behaviour (breeding pairs less social with each other and with their offspring) and increased agonism following the cage change from on to off exhibit (Glatston et al., 1984).

In addition to the presence or absence of visitors, the number of visitors appears to have a measureable effect on the behaviour of captive primate species (Birke, 2002, Chamove et al., 1988, Cooke and Schillaci, 2007, Fa, 1989, Glatston, 1984, Hosey and Druck, 1987, Kuhar, 2008, Mitchell et al., 1991, 1992, Skyner et al., 2004, Todd et al., 2006, Wells, 2005, Wood, 1998) and this variable is the most frequently reported in the literature. It is less clear whether there is a visitor density effect on other mammals commonly housed in zoos, due in part to the relatively few visitor effect studies carried out on non-primates. Some felids, for example, appear to show little or no behavioural response to visitors (Margulis et al., 2003, O’Donovan et al., 1993), whereas other studies report a visitor density influence on the behaviour of zoo- housed cats (Mallapur and Chellam, 2002, Sellinger and Ha, 2005). Ungulates have not been widely studied in this context, but sika deer (Cervus nippon; Shen-Jin et al., 2010) and Soemmerring's gazelle (Gazella soemmerringii; Mansour et al.2000) both show increased alertness as visitor density increases, and higher levels of aggression have been found in gaur (Bos gaurus gaurus; Sekar et al., 2008) and blackbuck (Antilope cervicapra; Rajagopal et al., 2011) when visitor pressure is high.

The behaviour of petting zoo animals in relation to zoo visitors has not been a common focus for visitor effect researchers but there are reports that the behaviour of ungulates housed in petting zoos can be affected by visitor density. African pygmy goats (Capra hircus) and Romanov sheep (Ovis aries) were significantly more likely to display "undesirable behaviour," such as head tossing, head butting, foot stamping, rearing, nose-blowing, and moving away, when the number of people within their enclosure was higher (Anderson et al., 2002), but this effect was not significant in a later study at the same petting 
zoo (Anderson et al., 2004). Furthermore, this later study identified higher rates of undesirable behaviour in association with increased levels of visitors touching the animals, whereas no relationship was found between these variables in the earlier study (Anderson et al., 2002).

Some of the difficulties in interpreting studies such as these can be helped by considering that the way in which animals respond to people is likely to be a consequence of their past history of interactions with people as well as their species (Hosey, 2008, 2013). These interactions can be positive as well as negative, and this raises the possibility that positive contact with humans could be enriching in some circumstances (Claxton, 2011). Increased contact time or structured activities with keepers have been shown to lead to changes in behaviour of the animals that have been interpreted as improving welfare in zoos (Baker, 2004, Carrasco et al., 2009, Manciocco et al., 2009) and laboratories (reviewed in Rennie and Buchanan-Smith, 2006). Zoo visitor effects on behaviour which have been interpreted as possibly enriching have usually involved feeding by the public, even in circumstances where this was not permitted by the zoo (Cook and Hosey, 1995, Fa, 1989). However, although there are reports of individual animals, such as parrots (Nimon and Dalziell, 1992), gorillas (Vrancken et al., 1990) and gibbons (Lukas et al., 2002), who actively solicited contact with the public, there appear to be no empirical studies, other than that of Anderson et al. (2002), of whether contact with zoo visitors in itself could be enriching for captive terrestrial mammals.

Here we present the results of two studies undertaken on a group of mixed-breed goats, llama, and Vietnamese pot-bellied pigs in a safari park petting zoo. Study 1 was an investigation to determine if the presence and density of visitors has an effect on the behaviour of these animals. It was predicted on the basis of the two studies by Anderson et al. $(2002,2004)$ that the presence and density of visitors would have a negative effect on the animals' behaviour. Study 2 was an experiment to test whether physical contact with zoo visitors results in behavioural changes in these animals which are consistent with the hypothesis that the contact is enriching for them. In the study by Anderson et al. (2002) human touches did not appear to be enriching for the animals, so in our study visitors were asked to groom the animals with a grooming tool instead, as this might provide greater tactile stimulation for the animals. 


\section{Methods}

\subsection{Study animals and setting}

The study animals consisted of 15 Angora, pygmy, and mixed-breed goats (Capra hircus ssp.), 16 llama (Llama glama), and six Vietnamese pot-bellied pigs (Sus scrofa) housed at the Pets Farm exhibit at Blair Drummond Safari and Adventure Park in Stirling, Scotland. All animals were captive born, included males and females and a range of ages. Five of the llama were born during the winter break, halfway through the study. The Pets Farm paddock also housed five greater rhea (Rhea americana) but the birds did not regularly interact with the study animals and were not part of the study. The Pets Farm exhibit is a large grassy paddock, approximately 0.4 ha in size, and is sparsely dotted with large trees (see Figure 1). A concrete path for visitors winds through the exhibit, but visitors were free to leave the path and approach the animals. No feeding of animals by visitors was permitted. The keepers provided the study animals with feeding troughs filled with hay as well as browse during the day. None of the observations for this study was collected when a Blair Drummond Safari park keeper was in the paddock. Observations were always suspended when keepers approached the paddock gate and were not resumed until the keeper had left the enclosure and the animals had stopped being alert to the keeper. A small area underneath one of the trees was fenced off to form a retreat area which visitors were prohibited from entering, but still allowed visitors to touch the animals. The retreat area was not accessible to the pigs because of the position of the fence rails, but the area was accessible to the goats and llama.

\subsection{Behavioural categories}

The same mutually exclusive behavioural categories were used for the goat, llama, and pigs

(Table 1). Several behavioural categories were added following the birth of five llama young. These behaviours, denoted with an asterisk (*), were not included in most statistical comparisons between conditions but were included in analyses of individual conditions where relevant. For Study 2 the additional categories 'tolerate grooming' and 'respond to grooming' were added, as well as several categories of human behaviour (Table 1). Visitor density was collected using the following categories: 110 visitors, $11-20$ visitors, $21-50$ visitors, $51-99$ visitors, 100 or more visitors. 


\subsection{Study 1: Data collection}

Observations in all conditions were made between the hours of 10:00 and 16:00 to ensure the data were not influenced by the time of day. Instantaneous scan samples (Martin and Bateson 2007) on five individuals per species were collected every ten minutes ensuring even distribution (mean 9.6 scans/day).

The species subgroups for each scan were selected systematically. The behaviour of the first five individuals of each of the study species observed was recorded, totalling 15 focal animals per scan; the beginning location of scans alternated from left to right, right to left, middle to left, and middle to right. This routine ensured that animals in all areas of the paddock were equally represented in the samples and active animals were not over-represented. Data were collected on a Psion Workabout using the behavioural software program The Observer (Noldus).

To obtain visitor presence and density data across seasons, two data collection periods were used. These periods were separated by the winter, during which the park was closed to the public. Observations were made with both visitors present (V) and visitors absent (NV) for both data collection periods. The schedule of observations, and number of scans collected are shown in Table 2.

The presence of the data collector (AF) during the NV conditions essentially constitutes an audience of one; although the effect of the presence of the researcher could not be eliminated, measures were taken to attempt to lessen the potential effect of observation. Upon arrival at Pets Farm for a sampling session, the researcher sat quietly in the middle of the field and waited to begin sampling until the animals habituated to her presence; habituation was considered to have taken place when the animals no longer visually monitored her or startled at her movements, usually about five minutes.

\subsection{Study 2: Procedure}

This study used the same animals as Study 1, although the llamas were excluded from statistical analysis as visitors only groomed them twice. Continuous focal animal samples (Martin and Bateson, 2007) were used to collect the data, which were collected with a Psion Workabout using the behavioural 
software program The Observer (Noldus). Observations for both 'no visitor grooming' (NVG; goat, $\mathrm{n}=46$ samples; pig, $\mathrm{n}=84$ samples) and 'visitor grooming' (VG; goat, $\mathrm{n}=38$ samples; pig, $\mathrm{n}=12$ samples) conditions were made simultaneously over a 19 day period in September-October 2004. To achieve the VG condition, participants were provided with a grooming tool, which was a solid rubber scrubbing block with long flexible teeth designed to groom domestic animals (Mikki ${ }^{\mathrm{TM}} 6275-185$, Dorking, Surrey, UK). NVG samples began when a visitor approached a study animal to within contact range (about $1 \mathrm{~m}$ ) and ended when either the animal or the visitor walked away, signalling an end to the interaction. VG samples began when visitors, wielding the grooming tool provided by the researcher, approached the study animals to within contact range and ended when either the visitor or animal walked away, indicating the interaction had come to an end. In some cases, during both the NVG and VG samples, the study animal walked or ran away from the approaching visitor before contact had taken place and either one of the following scenarios was then recorded for the interaction: 1) The behaviour Avoid Visitor was recorded and then the sample was ended if the visitor did not pursue the animal; or 2) The behaviour Avoid Visitor was recorded and the sample continued if the visitor pursued the animal. As in Study 1, none of the observations for this study was collected when a Blair Drummond Safari Park keeper was in the paddock.

\subsection{Statistical Analysis}

For Study 1, proportions were calculated using The Observer's Elementary Statistics feature and then exported to SPSS (version 15, 2006, Chicago) for further statistical analysis. The proportion of study animals per sample engaged in the behavioural categories collected were calculated and used in statistical testing as the unit of analysis. Randomisation tests as described by Todman and Dugard (2001) were used to compare the behaviour of the study animals and visitor density levels between conditions because of the small sample size and the non-normal distribution of the data. It was not possible to carry out a random assignment of treatments to observation periods or participants, which is a requirement of randomisation tests. However, it is argued that the timing of the conditions was essentially random with 
respect to animal behaviour (i.e. the introduction or withdrawal of visitors was not response-guided) and is, therefore, a valid use of this statistical technique. Design 1, a phase design, suitable for analysing data for a single case study was calculated to determine significant changes in animal behaviour between conditions. Each species was treated as a single participant because individuals were not identified and because the behaviour of socially-housed animals is not independent (Martin and Bateson, 2007). The test statistic for Design 1 was the difference between condition means. All tests were two-tailed, and the level of significance employed was 0.01 to reduce the risk of committing a Type 1 error when conducting multiple tests.

Descriptive statistics were used to compare the level of interaction with visitors between conditions. The mean rather than the median is reported because of the tendency of the median to equal zero. To determine if there was any relationship between the proportion of behaviours and the number of people in the Pets Farm paddock, correlation was used. The distribution of proportions was not normal and could not be satisfactorily transformed, therefore a non-parametric statistic, Spearman rank order correlation, was employed.

For Study 2, frequencies and durations of bouts were calculated using The Observer's Elementary Statistics feature and then exported to SPSS (version 15, 2006, Chicago) for further statistical analyses. Because the focal samples varied in length, depending on how long the focal animal interacted with visitors, frequencies were converted to frequency per hour before statistical analysis to achieve standardisation. The grooming experiment was consistent with an alternating design with random assignment of observation periods to treatment conditions, making Design 5a (small groups with two randomised treatments) as described in Todman and Dugard (2001) a suitable randomisation test for the data. The test statistic for Design 5a is the residual sum of squares.

\section{Results}

3.1 Potential Confounding Variables: Visitor Density and Weather 
Because two observation periods (Autumn and Spring) were utilised, separated by a period of zoo closure, potential differences between the two periods in visitor density and weather could be confounding variables. Visitor density between the two visitor presence conditions was compared using a randomisation test. There was no significant difference in visitor density between the two periods in which visitors were present (test statistic $=0.991, \mathrm{n}=251, p=0.548$ ). The weather did not vary greatly between visitor presence periods (Table 3) although there was more sunshine and less rain in Spring compared to Autumn.

\subsection{Study 1 The Effect of visitors on Petting zoo animals}

\subsubsection{The Effect of the Presence of Visitors on Behaviour}

The behaviour of the three species groups did not differ significantly between Autumn and Spring for the visitor presence condition. There was no change in goat or llama behaviour between the NV and V (Autumn and Spring combined) conditions, but the behaviour of the pigs was affected by whether members of the public were present within the Pets Farm enclosure. For the pigs, the following behaviours decreased significantly in the presence of visitors: Affiliate with Conspecifics (test statistic= $0.002, p=0.001$, proportion of total sample points= 0.001), Aggression between Conspecifics (test statistic $=-0.004, p=0.001$, proportion of total sample points $=0.002$ ), and Sit (test statistic $=-0.006, p=$ 0.001 , proportion of total sample points $=0.005)$.

\subsubsection{Comparison of Species Interactions with Visitors}

All three study species interacted with visitors in the visitor presence conditions. The goats exhibited Contact With Visitors in $12 \%$ of the scans, the pigs slightly less at $9 \%$, and the llama in $4 \%$ of scans. The llama and goats avoided visitors in $1 \%$ of scans, while the pigs avoided visitors in less than $1 \%$ of scans. Neither the goats nor the pigs directed aggression towards visitors, but the llama spent $0.1 \%$ of scans engaged in visitor-directed aggression. 


\subsubsection{The Relationship between Visitor Density and Behaviour}

The relationship between visitor density and behaviour was analysed for all three species. The proportion of Contact With Visitors increased significantly in the goat group as the numbers of visitors increased (Autumn: $\mathrm{r}=0.327, p=0.001, \mathrm{n}=125$; Spring: $\mathrm{r}=0.427, p=0.001, \mathrm{n}=126$ ), while Proximity With Non-conspecifics $(\mathrm{r}=-0.257, p=0.004, \mathrm{n}=126)$ and Contact With Conspecifics $(\mathrm{r}=-0.231, p=0.009$, $\mathrm{n}=126$ ) decreased significantly as visitor density increased in the Spring (no data were collected for these

behaviours in Autumn). Only one llama behaviour was dependent on the level of visitor density, with the proportion of Contact With Visitors increasing significantly as visitor numbers increased (Autumn: $\mathrm{r}=$ $0.364, p=0.001, \mathrm{n}=126$; Spring: $\mathrm{r}=0.283, p=0.001, \mathrm{n}=126$ ). Two pig behaviours were dependent on visitor density. The proportion of Contact With Visitors increased significantly as visitor density increased (Autumn: $\mathrm{r}=0.486, p=0.001, \mathrm{n}=125$; Spring: $\mathrm{r}=0.344, p=0.001, \mathrm{n}=126$ ). The proportion of Feed was only significantly negatively correlated with visitor density in the Autumn $(\mathrm{r}=-0.361, p=0.001$, $n=125)$.

\subsection{Study 2: The Effects of Visitor Grooming of Animals}

There was no significant difference in visitor density between VG and NVG conditions for either the goat (test statistic $=0.423, \mathrm{p}=0.033, \mathrm{n}=119$ ) or pig (test statistic $=0.571, \mathrm{p}=0.189, \mathrm{n}=47$ ) groups. There were no significant differences in any of the animal behaviours between VG and NVG conditions for either goats or pigs. The duration of Tolerate Grooming was significantly greater than Respond To Grooming for both goats (test statistic $=25.44, \mathrm{p}=0.001, \mathrm{n}=110$; Fig 2a) and pigs (test statistic $=28.25, \mathrm{p}=0.001$, $\mathrm{n}=15$; Fig 2b), but the frequency of Tolerate Grooming was only significantly different for goats (test statistic $=1.28, p=0.005, n=110 ;$ Fig $2 \mathrm{c}$ ). Visitor density was significantly negatively correlated with both frequency and duration of Respond To Grooming in goats (frequency: $\mathrm{r}=-0.315, \mathrm{p}=0.004, \mathrm{n}=78$; duration: $r=-0.319, p=0.004, n=78)$, but there was no association between these variables in the pigs. 
Visitors groomed goats significantly more frequently and for significantly longer than they interacted with them without grooming, as shown by the comparison of Groom Animal in the VG with Affiliate With Animal in the NVG condition (duration: test statistic $=24.39, \mathrm{p}=0.001, \mathrm{n}=155$; frequency: test statistic= 0.018, $\mathrm{p}=0.006, \mathrm{n}=155$; Fig 3a and $\mathrm{b}$ ), but no such difference was found for the pigs.

\section{Discussion}

\subsection{Visitor Density and Weather are Unlikely to be Confounding Variables}

The comparison of visitor density between the Autumn and Spring samples demonstrates a consistent level of visitors to the petting zoo between the pre- and post winter break conditions. Weather conditions were also very similar, suggesting different levels of visitor density and weather were not differentially affecting the behaviour of the study animals between these two periods. Collecting data on potential confounding variables is particularly important in visitor effect research because of the difficulty controlling visitor-related variables, such as density, in the zoo environment. These results also suggest that a sustained period without the presence of visitors (i.e. the winter closure of the zoo) does not lead to an increased visitor effect once visitor-related stimuli are re-introduced, and demonstrates that the removal and reintroduction of visitor-related stimuli is not necessarily a welfare concern for animals intermittently exposed to zoo visitors.

\subsubsection{The Presence of Visitors Affects Vietnamese Pot-bellied Pig Behaviour}

The hypothesis that the behaviour of the three study species would be negatively affected by the presence of visitors was only supported in one of the three study species, the pigs. Goat and llama behaviour was unaffected by the presence of visitors, a finding which suggests that these species do not experience a visitor effect when housed in these conditions. This result is in keeping with reports that African pygmy goats exhibited less undesirable visitor-directed behaviour ( 0.15 undesirable behaviours

per hour) than Romanov sheep (1.68 undesirable behaviours per hour; Anderson et al., 2002), which was interpreted by those authors to indicate that the goats were less fearful of people than the sheep. Our 
study demonstrated a negative visitor effect in the pigs. The level of expression of the affected behaviours, however, was low and the behavioural change was likely to have a negligible effect on their welfare. Given that all three significantly affected behaviours decreased in the presence of visitors, it is also possible that the proportion of behaviours decreased simply due to an increase in the number of behaviours performed in this condition (i.e. the proportion of pigs sitting decreased because they were also now interacting with visitors).

The decreased inactivity in the pigs in this study is similar to findings of increased activity under conditions of greater visitor pressure in zoo-housed gaur (Sekar et al., 2008), and blackbuck (Rajagopal et al., 2011). Conversely both of these species, and also gazelles (Mansour et al., 2000), showed higher aggression under the same conditions, whereas the pigs in this study showed lower aggression. These observed behavioural changes related to visitor pressure in the pigs are surprising given that Vietnamese pot-bellied pigs are a breed frequently kept as household pets and, therefore, might be assumed to be less susceptible to the influence of the presence of visitors than non-companion animal species such as llama and goats. Overall we conclude that the welfare of Vietnamese pot-bellied pigs and llama should be carefully monitored as they are more sensitive to visitor pressure than goats.

\subsubsection{Species Differences in Interactions with Visitors}

Although there may be breed differences in the temperament of goats, we did not discriminate between breeds. Our findings that mixed-breed goats had high levels of interactions with vistiors is not unexpected given the previous reports which indicate that African pygmy goats are less fearful of zoo visitors than sheep (Anderson et al., 2002, 2004). Llama aggression towards visitors suggests that these ungulates may be slightly less tolerant of contact with visitors than goats. Indeed llama are often used as guard animals for sheep producers given their predisposition to be aggressive to sheep threatening-canids (Cavalcanti and Knowlton, 1998).Further research on the suitability of llama in petting zoos is needed.

While the aggression displayed by the pigs was not directed toward visitors, the change in their behaviour when visitors were present suggests that Vietnamese pot-bellied pigs experience more visitor pressure than petting zoo-housed goats. 


\subsubsection{Visitor Density Effects}

An association between visitor density and species behaviours was found in all three species groups, but not always in the direction predicted. The strength of the positive association, based on essentially equal sample sizes, between study species-visitor contacts and visitor density was greater in the Spring visitor condition for the goats but less strong in the Spring for the llama and pigs. While the birth of the llama young in the Spring might explain the reticence of the llama to interact with visitors, it is also possible that the winter break contributed to the decline in the willingness to interact with humans in the presence of a larger number of zoo visitors. The association between contact with visitors and visitor density in this study contradicts previous reports of increased rates of undesirable visitor-directed goat and sheep behaviour as the number of people increased (Anderson et al., 2002).

The change in goat group cohesion in association with visitor density suggests that visitor density can have an effect on the instinct to herd in ungulates. This result is concordant with Thompson's (1989) suggestion that the increased vigilance that she observed in twelve species of zoo-housed ungulates in response to both visitors and keepers reflected a decrease in group cohesion as a result of the animals' enhanced curiosity towards people. Feeding behaviour in the Pets Farm goats, which represents a large proportion of ungulate activity budgets (49\% for feral goats: Stronge et al., 1997), was not affected by visitor density and suggests that visitor density may not have an intense effect on goats housed in petting zoos. Pig feeding behaviour was only significantly affected in the Autumn, although there was a trend in the Spring $(\mathrm{p}<0.05)$. Visitor density has also been associated with reduction in feeding and foraging behaviour in park sika deer (Shen-Jin et al., 2010). Overall, the results of the visitor density analysis suggest that there are species differences in the degree of influence this visitor-related variable has on the behaviour of petting zoo-housed animals. In addition, these results indicate that high visitor density may not necessarily decrease the educational and entertainment value of such exhibits for zoo visitors, as has been previously suggested (Anderson et al., 2002, 2004).

\subsection{Is Grooming by Visitors Enriching for the Animals?}


The introduction of visitor grooming did not affect the behaviour of the goats or the Vietnamese pot-bellied pigs, suggesting that the technique was not enriching to these animals. The lack of change in avoidance of visitors suggests that grooming visitors were not perceived as more threatening by the petting zoo inhabitants than visitors who did not groom them.

The lack of a significant response to grooming has several explanations. It could be that goats and pigs simply did not enjoy being groomed. This explanation seems unlikely because during a pilot test two of the authors elicited responses to grooming that suggested sensory enrichment was taking place. It is also possible that the grooming tool was not ideal for this task, although this also seems unlikely as it was a commercially available product designed for use on domestic animals, and was the same tool used in the pilot test.

The most plausible explanation for the ineffectiveness of the visitor grooming enrichment is the incorporation of visitors in dispensing the enrichment. The large number of unfamiliar visitors participating in the experiment adversely affected the goats' response to grooming and this was evidenced by the significant negative correlation between Respond To Groom and visitor density. The pigs' response to grooming was not dependent on visitor density, and indicates that grooming may not be an effective method of improving human animal relationships in petting zoo pigs.

It also seems likely that visitor inexperience in grooming domestic animals was, in part, responsible for the ineffectiveness of the grooming. Given the anecdotal results of the pilot test in which experienced yet unfamiliar animal behaviour researchers were able to elicit positive responses to grooming, it appears that visitors might have dispensed more effective grooming enrichment if they were educated on proper grooming techniques. During the experimental trials, visitors were given general instructions by the researcher on how to groom the animals, but were not directed further. A few visitors asked the researcher for more direction in proper grooming methods and received instruction. The data on gentling in farm animals, in which stockpersons handle the animals, also suggests that experienced animal handlers may be more effective in creating a positive human animal relationship (Hargreaves and Hutson, 1990, Lensink et al., 2000). 
The lack of visitor grooming experience was anticipated to be a potential factor in the effectiveness of the grooming experiment, but it was decided to test the enrichment on the essentially untrained public because the visitor-friendly grooming method was developed in part to provide some relief for keepers, who provide the majority of the enrichment captive animals receive. The role visitor skill plays in this enrichment technique could be moderated by providing instruction signs, although this might not be the best solution in the petting zoo environment where many of the visitors grooming will be children who are unable or unwilling to read the instructions before they make contact with the animals. A better, but more labour-intensive solution may be to have daily keeper grooming sessions which would give visitors hands on instruction in proper grooming techniques.

The failure of visitor grooming to provide enrichment for the petting zoo animals did not prevent grooming from positively affecting visitor behaviour. The frequency per hour and the duration of visitor interactions with the petting zoo goats increased significantly in the grooming condition, suggesting that visitor interest in non-aggressive contact with the goats increased during the enrichment condition. Surprisingly, the increased frequency and duration of contact with goats in the grooming condition did not extend to the pigs, suggesting visitors found the pigs less rewarding to groom. Despite the increased interest in interacting with the petting zoo goats, visitors did not pursue or chase the goats or pigs any more frequently in the grooming condition, which was anticipated to be a potential negative consequence of encouraging visitors to take part in increased contact with the animals. The general lack of visitor illtreatment of the animals in both experiments bodes well for further attempts to incorporate visitors into animal enrichment programmes.

The surprisingly low level of harassment or aggression visitors directed towards the study animals is noteworthy and is likely to be a factor in the study animals' ability to cope with the presence of visitors. It also suggests that moderating visitor behaviour, in addition to providing retreat areas (Anderson et al., 2002) and other kinds of environmental enrichment for the animals, may be effective when attempting to reduce the negative impact of zoo visitors on the welfare of petting zoo-housed animals. 


\section{Conclusion}

The data indicate that mixed-breed goats, llama, and Vietnamese pot-bellied pigs exhibit a visitor effect in varying degrees, but the quantitative and qualitative nature of the behavioural changes suggest that the three study species were relatively unaffected by the presence of visitors. Considering the petting zoo environment within the visitor effect literature and its accompanying methodological concerns may also be helpful when interpreting the data on behavioural changes associated with visitor-related variables. Overall, this study provides evidence that visitors do not necessarily have an extensive impact on petting zoo goat, llama, and pig behaviour and may not decrease their welfare when housed under these conditions. However, it also suggests that the opportunity to interact with people is not in itself enriching for these animals.

\section{Conflict of interest}

The authors of this manuscript have no conflict of interest that would inappropriately bias this research.

\section{Acknowledgements}

The authors would like to extend their thanks to Blair Drummond Safari Park for assisting with this project. A. Farrand received a University of Stirling studentship which funded this study and she is grateful for their support. Statistical advice from Dr. Amy Plowman, Prof. Graham Ruxton, Dr. Pat Dugard, and Dr. John Todman was invaluable. The research was approved by the University of Stirling Psychology Ethics Committee and adhered to the legal requirements in the United Kingdom.

\section{References}

Anderson, U.S., Benne, M., Bloomsmith, M.A. \& Maple, T.L. 2002. Retreat space and human visitor density moderate undesirable behavior in petting zoo animals. J. Appl. Anim. Welfare Sci. 5, 125137. 
Anderson, U.S., Maple, T.L. \& Bloomsmith, M.A. 2004. A close keeper-nonhuman animal distance does not reduce undesirable behavior in contact yards goats and sheep. J. Appl. Anim. Welfare Sci. 7, 59-69.

Baker, K.C. 2004. Benefits of positive human interaction for socially housed chimpanzees. Anim. Welfare 13, 239-245.

Birke, L. 2002. Effects of browse, human visitors and noise on the behaviour of captive orangutans. Anim. Welfare 11, 189-202.

Blaney, E.C. \& Wells, D.L. 2004. The influences of a camouflage net barrier on the behaviour, welfare, and public perceptions of zoo-housed gorillas. Anim. Welfare 13, 111-118.

Carlstead, K. \& Brown, J.L. 2005. Relationships between patterns of fecal corticoid excretion and behavior, reproduction, and environmental factors in captive black (Diceros bicornis) and white (Ceratotherium simum) rhinoceros. Zoo Biol. 24, 215-232.

Carrasco, L., Colell, M., Calvo, M., Abelló, M.T., Velasco, M. \& Posada, S. 2009. Benefits of training/playing therapy in a group of captive lowland gorillas (Gorilla gorilla gorilla). Anim. Welfare 18, 9-19.

Cavalcanti, S.M.C. \& Knowlton, F.F. 1998. Evaluation of physical and behavioral traits of llamas associated with aggressiveness toward sheep-threatening canids. Appl. Anim. Behav. Sci. 61, 143158.

Chamove, A., Hosey, G.R. \& Schaetzel, P. 1988. Visitors excite primates in zoos. Zoo Biol. 7, 359-369.

Claxton, A.M. 2011. The potential of the human-animal relationship as an environmental enrichment for the welfare of zoo-housed animals. Appl. Anim. Behav. Sci. 133: 1-10.

Cook, S. \& Hosey, G.R. 1995. Interaction sequences between chimpanzees and human visitors at the zoo. Zoo Biol. 14, 431-440.

Cooke, C.M. \& Schillaci, M.A. 2007. Behavioral responses to the zoo environment by white handed gibbons. Appl. Anim. Behav. Sci. 106, 125-133. 
Davis, N., Schaffner, C.M. \& Smith, T.E. 2005. Evidence that zoo visitors influence HPA activity in spider monkeys (Ateles geoffroyii rufiventris). Appl. Anim. Behav. Sci. 90, 131-141.

Fa, J.E. 1989. Influence of people on the behavior of display primates. In: Segal, E.F. (Ed). Housing, Care, and Psychological Well-being of Captive and Laboratory Primates. Noyes Publications, Park Ridge, USA, pp. 270-290.

Glatston, A.R., Geilvoet-Soeteman, E., Hora-Pecek, E., \& van Hooff, J.A.R.A.M. 1984. The influence of the zoo environment on social behavior of groups of cotton-top tamarins, Saguinus oedipus oedipus. Zoo Biol. 3, 241-253.

Hargreaves, A.L. \& Hutson, G.D. 1990. The effect of gentling on heart rate, flight distance and aversion of sheep to a handling procedure. Appl. Anim. Behav. Sci. 26: 243-252.

Hosey, G. 2000. Zoo animals and their human audiences: What is the visitor effect? Anim. Welfare 9, 343-357.

Hosey, G. 2008. A preliminary model of human-animal relationships in the zoo. Appl. Anim. Behav. Sci. $109,105-127$.

Hosey, G. 2013. Hediger revisited: how do zoo animals see us? J. Appl. Anim. Welfare Sci. 16, in press.

Hosey, G. \& Druck, P.L. 1987. The influence of zoo visitors on the behaviour of captive primates. Appl. Anim. Behav. Sci. 18, 19-29.

Jackson, K.M.A. \& Hackett, D. 2007. A note: the effects of human handling on heart girth, behavior and milk quality in dairy goats. Appl. Anim. Behav. Sci. 108: 332-336.

Kuhar, C.W. 2008. Group differences in captive gorillas' reaction to large crowds. Appl. Anim. Behav. Sci. $110,377-385$.

Lensink, B.J., Fernandez, X., Boivin, X., Pradel, P., LeNeindre, P. \& Veissier, I. 2000. The impact of gentle contacts on ease of handling, welfare, and growth of calves and on quality of veal meat. J. Anim. Sci. 78: 1219-1226. 
Lukas, K.E., Barkauskas, R.T., Maher, S.A., Jacobs, B.A., Bauman, J.E., Henderson, J.A. \& Calcagno, J.M. 2002. Longitudinal study of delayed reproductive success in a pair of white-cheeked gibbons (Hylobates leucogenys). Zoo Biol. 21, 413-434.

Mallapur, A. \& Chellam, R. 2002. Environmental influences on the activity budget of leopards (Panthera pardus) in four zoos in southern India. J. Appl. Anim. Welfare Sci. 5, 111-124.

Mallapur, A., Sinha, A. \& Waran, N. 2005. Influence of visitor presence on the behaviour of captive liontailed macaques (Macaca silenus) housed in Indian zoos. Appl. Anim. Behav. Sci. 94, 341-352.

Manciocco, A., Chiarotti, F. \& Vitale, A. 2009. Effects of positive interaction with caretakers on the behaviour of socially housed marmosets (Callithrix jacchus). Appl. Anim. Behav. Sci. 120, 100107.

Mansour, A.A.H., Zakaria, A-H. \& Fraser, A.F. 2000. Effect of enclosure quality on reactivity and welfare of captive Soemmerring's gazelle (Gazella soemmerringii). J. Appl. Anim. Welfare Sci. 3: 335-343.

Margulis, S.W., Hoyos, C. H. \& Anderson, M. 2003. Effect of felid activity on zoo visitor interest. Zoo Biol. 22, 587-599.

Markowitz, T.M., Dally, M.R., Gursky, K. \& Price, E.O. 1998. Early handling increases lamb affinity for humans. Anim. Behav. 55: 573-587.

Martin, P. \& Bateson, P. 2007. Measuring Behaviour: An introductory guide. Cambridge University Press, Cambridge UK, $3^{\text {rd }}$ edition.

Mitchell G., Herring, F., Obradovich, S., Tromborg, C., Dowd, B., Neville, L.E., \& Field, L. 1991. Effects of visitors and cage changes on the behaviour of mangabeys. Zoo Biol. 10, 417-423.

Mitchell, G., Tromborg, C.T., Kaufman, J., Bargabus, S., Simoni, R. \& Geissler, V. 1992. More on the "influence" of zoo visitors on the behaviour of captive primates. Appl. Anim. Behav. Sci. 35, 189198. 
Nimon, A.J. \& Dalziel, F.R. 1992. Cross-species interaction and communication: a study method applied to captive siamang (Hylobates syndactylus) and long-billed corella (Cacatua tenuirostris) contacts with humans. Appl. Anim. Behav. Sci. 33, 261-272.

O’Donovan, D., Hindle, J.E., McKeown, S. \& O’Donovan, S. 1993. Effect of visitors on the behaviour of female cheetahs. Int. Zoo Ybk. 32, 238-244.

Rajagopal, T., Archunan, G. \& Sekar, M. 2011. Impact of zoo visitors on the fecal cortisol levels and behavior of an endangered species: Indian blackbuck (Antelope cervicapra L.). J. Appl. Anim. Welfare Sci. 14: 18-32.

Rennie, A.E., \& Buchanan-Smith, H.M. 2006. Refinement of the use of non-human primates in scientific research. Part I: The influence of humans. Anim. Welfare 15: 203-213.

Rushen, J., Taylor, A.A. \& de Passillé, A.M. 1999. Domestic animals' fear of humans and its effect on their welfare. Appl. Anim. Behav. Sci. 65: 285-303.

Sellinger, R.L. \& Ha, J. C. 2005. The effects of visitor density and intensity on the behaviour of two captive jaguars (Panthera onca). J. Appl. Anim. Welfare Sci. 8, 233-244.

Sekar, M., Archunan, G. \& Rajagopal, T. 2008. Influence of zoo visitor presence on the behavior of captive Indian gaur (Bos gaurus gaurus) in a zoological park. J. Appl. Anim. Welfare Sci. 11: 352357.

Shen-Jin, L., Todd, P.A., Yan, Y., Lin, Y., Hongmei, F. \& Wan-Hong, W. 2010. The effects of visitor density on sika deer (Cervus nippon) behavior in Zhu-Yu-Wan Park, China. Anim. Welfare 19: 6165.

Skyner, L., Amory, J.R. \& Hosey, G. 2004. The effect of visitors on the self-injurious behaviour of a male pileated gibbon (Hylobates pileatus). Zool. Gart. 74, 38-41.

Stronge, D.C., Fordham, R.A. \& Minot, E.O. 1997. The foraging ecology of feral goats Capra hircus in the Mahoenui Giant Weta Reserve, Southern King Country, New Zealand. N. Z. J. Ecol. 21, 81-88.

Thompson, V.D. 1989. Behavioral response of 12 ungulate species in captivity to the presence of humans. Zoo Biol. 8, 275-297. 
Todd, P.A., Macdonald, C. \& Coleman, D. 2006. Visitor-associated variation in captive Diana monkey (Cercopithecus diana diana) behaviour. Appl. Anim. Behav. Sci. 107, 162-165.

Todman, J.B. \& Dugard, P. 2001. Single-case and small-n experimental designs. Lawrence Erlbaum Associates Inc., Mahwah, NJ, USA.

Wells, D.L. 2005. A note on the influence of visitors on the behaviour and welfare of zoo-housed gorillas. Appl. Anim. Behav. Sci. 93, 13-17.

Wood, W. 1998. Interactions among environmental enrichment, viewing crowds, and zoo chimpanzees (Pan troglodytes). Zoo Biol. 17, 211-230. 


\section{Figures}

Figure 1 Photo of the pets farm paddock at Blair Drummond Safari Park, showing the retreat area.

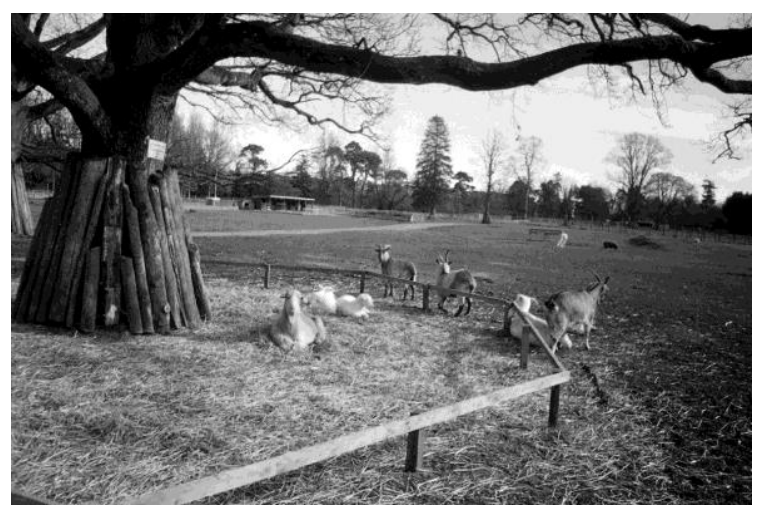


Figure 2a The difference in the median duration of Respond To Grooming and the median duration of Tolerate Grooming in the visitor grooming condition in the goat group.
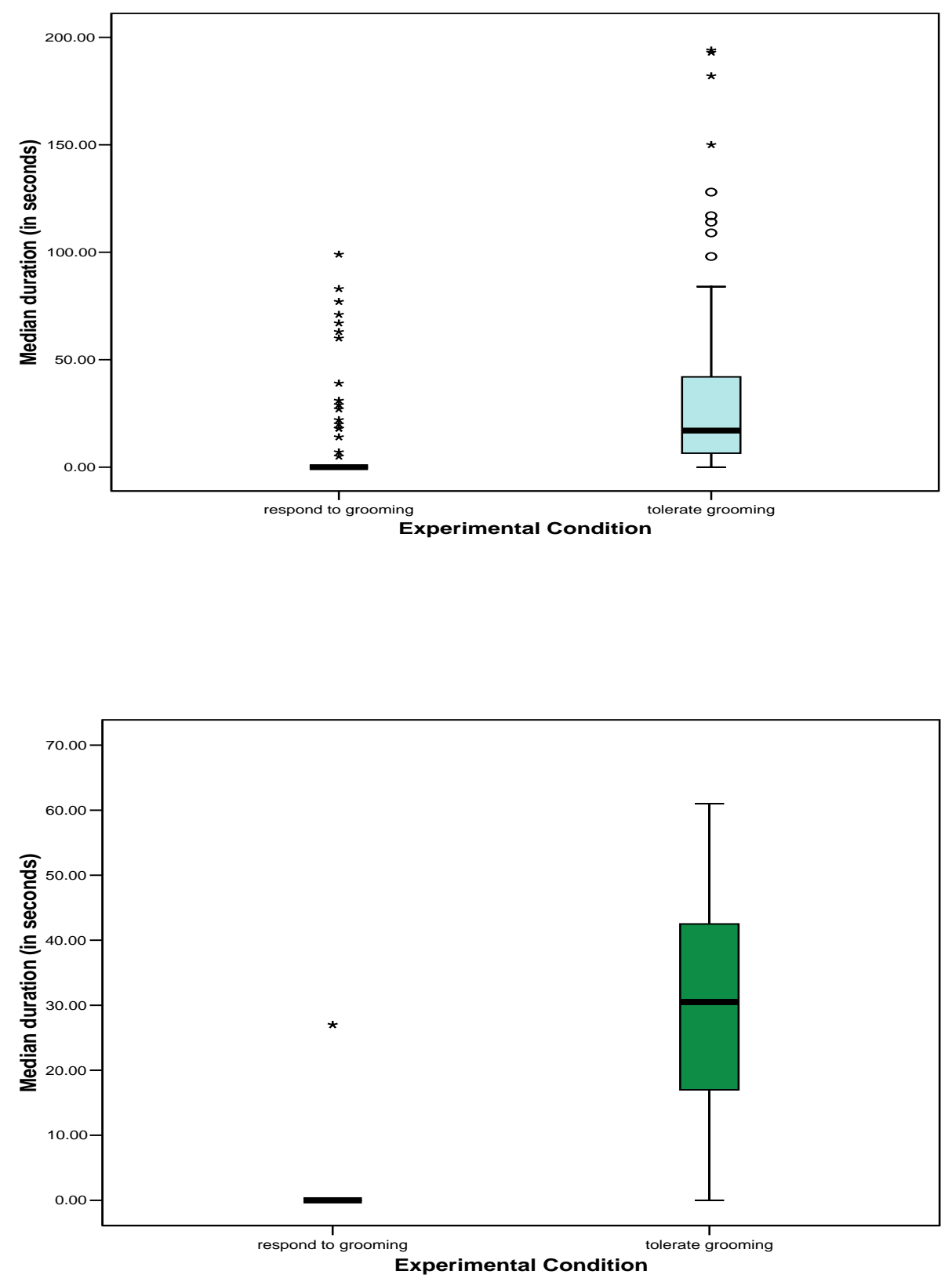

Figure 2b The difference between the median duration of Respond To Grooming and the median duration of Tolerate Grooming in the visitor grooming condition in the pig group. 


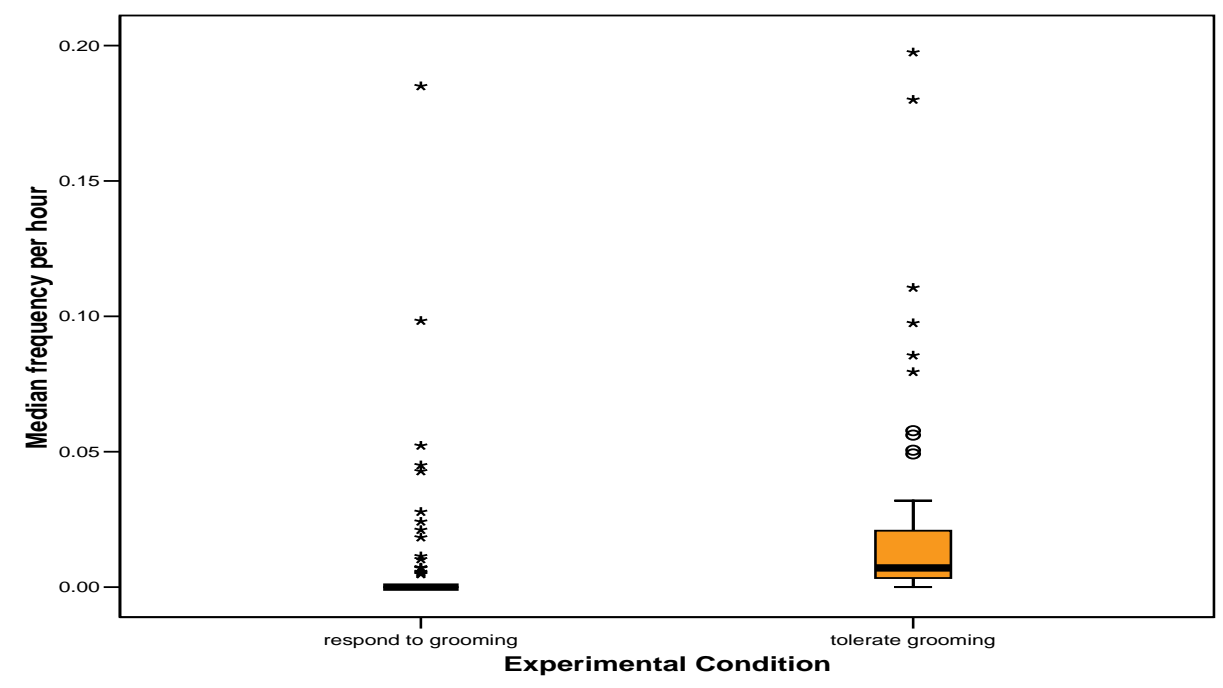

Figure 2c The difference in the median frequency per hour of Respond To Grooming and the median frequency per hour of Tolerate Grooming in the visitor grooming condition in the goat group. 


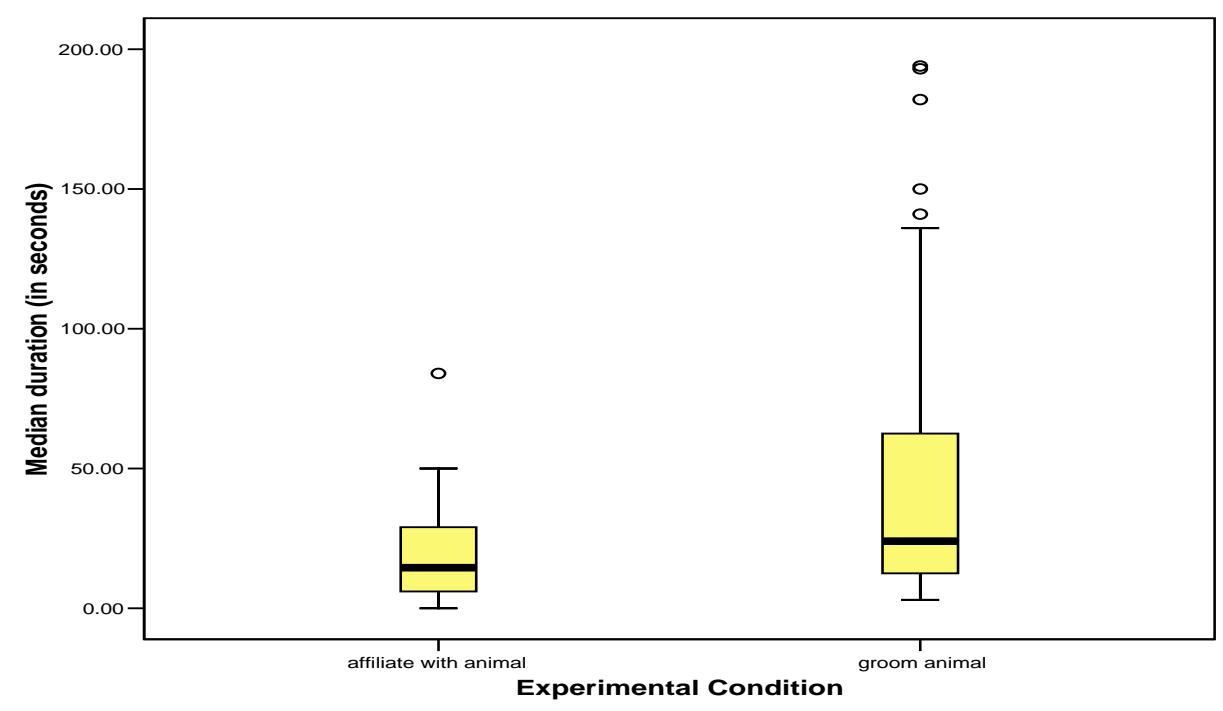

Figure 3a The difference in the median duration of Affiliate With Animal in the no visitor grooming condition compared to the median duration of Groom Animal in the visitor grooming condition directed toward the goat group.

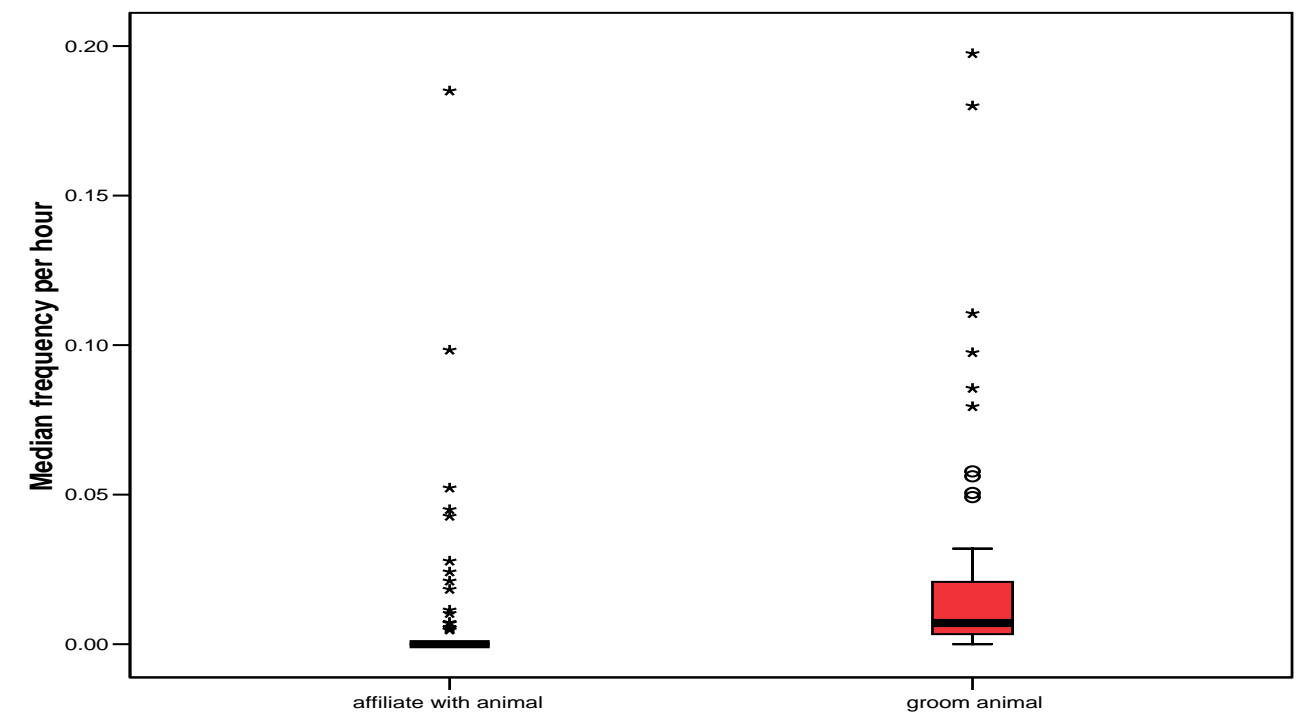

Figure 3b The difference in the median frequency per hour of Affiliate With Animal in the no visitor grooming condition compared to the median frequency per hour of Groom Animal in the visitor grooming condition directed toward the goat group. 